\title{
Multiperson Tracking With a Network of Ultrawideband Radar Sensors Based on Gaussian Mixture PHD Filters
}

\author{
Berk Gulmezoglu, Student Member, IEEE, Mehmet Burak Guldogan, Member, IEEE, \\ and Sinan Gezici, Senior Member, IEEE
}

\begin{abstract}
In this paper, we investigate the use of Gaussian mixture probability hypothesis density filters for multiple person tracking using ultrawideband (UWB) radar sensors in an indoor environment. An experimental setup consisting of a network of UWB radar sensors and a computer is designed, and a new detection algorithm is proposed. The results of this experimental proof-of-concept study show that it is possible to accurately track multiple targets using a UWB radar sensor network in indoor environments based on the proposed approach.
\end{abstract}

Index Terms-Multiple person detection, target tracking, PHD filter, ultra-wideband, radar.

\section{INTRODUCTION}

$\mathbf{W}$ IRELESS sensor networks (WSN) have received tremendous attention in last decade due to their critical importance in a wide variety of applications such as surveillance, and due to the theoretical and practical challenges they introduce [1], [2]. For indoor scenarios, ultrawideband (UWB) sensors can be employed because of their extraordinary resolution and localization precision [3], [4]. There are also additional advantages of UWB signals such as low power consumption, low probability of interception, and co-existence with a large number of devices [5]. For multisensor multi-object tracking applications, UWB is a wellsuited technology. Since UWB signals are characterized by the transmission of a few nanosecond duration pulses [6]-[9], they have very high time resolution and localization precision, which make UWB sensors an ideal equipment for short range radar sensor network applications [10], [11]. In this study, UWB radar sensors are employed for detecting and tracking

Manuscript received August 1, 2014; revised November 6, 2014; accepted November 8, 2014. Date of publication November 20, 2014; date of current version February 5, 2015. This work was supported in part by the Turk Telekom under Project 3015-02, and in part by the European Commission within the framework of the FP7 Network of Excellence in Wireless COMmunications \# under Contract 318306. The work of S. Gezici was supported by the Distinguished Young Scientist Award through the Turkish Academy of Sciences under Grant TUBA-GEBIP 2013. The associate editor coordinating the review of this paper and approving it for publication was Dr. Lorenzo Lo Monte.

B. Gulmezoglu is with the Department of Electrical and Computer Engineering, Worcester Polytechnic Institute, Worcester, MA 01609 USA (e-mail: bgulmezoglu@wpi.edu).

M. B. Guldogan is with the Department of Electrical and Electronics Engineering, Turgut Ozal University, Ankara 06010, Turkey (e-mail: bguldogan@turgutozal.edu.tr).

S. Gezici is with the Department of Electrical and Electronics Engineering, Bilkent University, Ankara 06800, Turkey (e-mail: gezici@ee.bilkent.edu.tr).

Color versions of one or more of the figures in this paper are available online at http://ieeexplore.ieee.org.

Digital Object Identifier 10.1109/JSEN.2014.2372312 multiple moving objects in an indoor environment in the context of passive localization [12]-[17].

Multiple target tracking is a subfield of signal processing with applications spanning many different engineering disciplines [18]. In this subfield of signal processing, the random finite set (RFS) approach is one of the newest developments that provides a general systematic framework for multi-target systems by modeling the multi-target state as an RFS [19], [20]. The RFS approach is considered to be a very promising alternative to handle the multi-target multidetection association problem faced in multi-target tracking applications. The RFS approach treats the collection of individual measurements and the individual targets as a set-valued measurement and set-valued state, respectively. It is shown that the sequential estimation of multiple targets buried in clutter with association uncertainties can be formulized in a Bayesian filtering framework by modeling set-valued measurements and set-valued states as RFSs [19]. The probability hypothesis density (PHD) filter, an approximation of this theoretically optimal approach to multi-target tracking, propagates the firstorder statistical moment of the RFS of states in time and avoids the combinatorial data association problem. The dimension of the PHD filtering is equal to the dimension of the single target state. Despite its advantages, the recursions of the PHD filter involve multiple integrals having no closed form solutions. There are two implementations of the PHD filter; one is using sequential Monte-Carlo (SMC) method, and the other one is using Gaussian mixtures (GM). Each implementation method has its own pros and cons [19]. GM implementation is very popular since it provides a closed form analytic solution to PHD recursions under linear Gaussian target dynamics and measurement models [19]. Moreover, contrary to SMC implementation, GM implementation provides reliable state estimates extracted from the posterior intensity in an easier and efficient way. Alternatively, SMC implementation imposes no such restrictions and has the ability to handle nonlinear target dynamics and measurement models. It can be said that SMC implementation is a more general framework for PHD recursions. On the other hand, its performance is affected by different kind of problems in reality [21]-[23]. Therefore, in general, the GM based approach is easier, effective and more intuitive.

Multiple target tracking via UWB sensors has been considered in some studies in the literature. In [24], time of flight information of the targets is used for tracking via PHD filters. 
A single scenario with targets moving in a straight line (no maneuvers) is considered, and directional horn antennas are used for powerful signal reception. Each sensor is equipped with one transmitter and two receivers, which are synchronized via a digital resonance oscillator. The blind zone problem and its solution are explained in [25] and new approaches are developed for this problem in tracking. In [26], multiple person tracking via UWB radar sensors is performed by utilizing time variations of the channel impulse response due to the presence of people between the transmitter and the receiver. Background subtraction and constant false alarm rate (CFAR) algorithms are employed for person detection, and GM-PHD filter is used for tracking. In a similar study, [27] proposes an indoor UWB person detection and ranging technique that does not require any information about the environment and exploits the temporal variations in the received signal due to the presence of a person. Finally, in [28], localization of a passive reflector based on backscattering range measurements is studied, and theoretical performance bounds are presented.

In this paper, a new approach is developed for multitarget tracking via a network of UWB radar sensors based on GM-PHD filtering. A novel detection technique is proposed for removing a significant part of clutter, which facilitates robust localization. The performance of the proposed tracking method shows that multiple targets can be tracked efficiently in an indoor environment. Although the PHD filtering approach has been considered for multi-target tracking in [24], the considered system has high cost and complexity due to the use of six experimental sensors, each equipped with one transmitter and two receivers, which employ directional horn antennas. Also, a single scenario is considered with targets moving in a straight line without any maneuvers [24]. In our study, four small off-the-shelf UWB radar sensors produced by TimeDomain [29] are employed, where each sensor has a single transmitter and a receiver. Scenarios containing multiple maneuvering targets are also investigated. In addition, the proposed approach does not make any specific assumptions about the environment and positions of the targets. To sum up, there are two main contributions in this paper: Firstly, we propose a new detection technique which effectively handles severe multipath. Secondly, the GM-PHD filter is successfully used in the tagless multi-person tracking problem using off-the-shelf UWB radar sensors.

The remainder of the manuscript is organized as follows: In Section II, sensor and measurement models are presented, and the proposed detection method is examined in Section III. Section IV presents the RFS based filtering. The details of the radar sensors are explained in Section V and Section VI discuses the experimental results. Finally, conclusions are given in Section VII.

\section{Sensor And Measurement Model}

Before describing the sensor and measurement models, the transmitted signal model for the UWB system is given first:

$$
s(t)=\sum_{j=0}^{N_{f-1}} \sum_{i=1}^{N_{p}} p\left(t-i T_{p}-j T_{f}\right)
$$

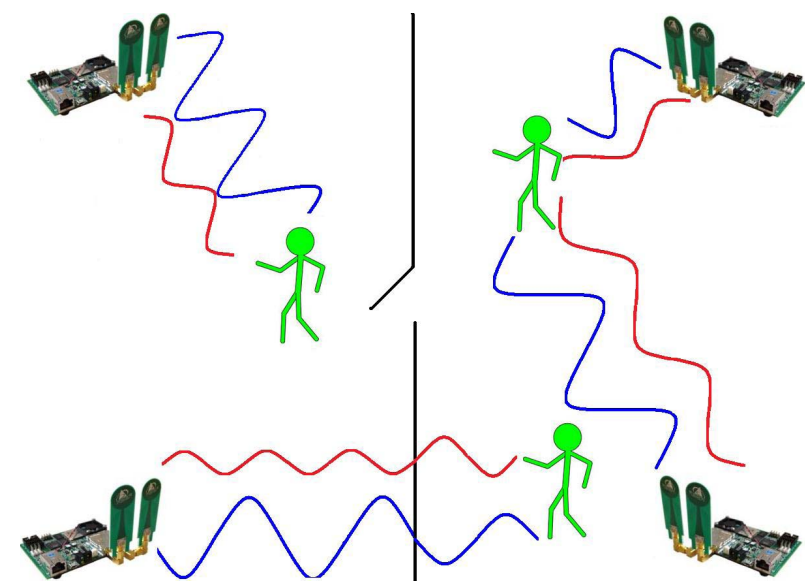

Fig. 1. Indoor environment with four UWB radar sensors. Blue signals and red signals represent transmitted and received signals, respectively.

where $p(t)$ represents the UWB pulse, $T_{f}$ is the duration of a frame, $T_{p}$ is the duration between UWB pulses in a frame (which is larger than the pulse duration), $N_{f}$ is the number of frames, and $N_{p}$ is the number of pulses in a frame. Signal $s(t)$ is produced by a UWB transmitter and the reflected signals are collected by a UWB receiver to determine the distances between targets and sensors in an indoor environment. In the process, time-of-arrival (ToA) parameters are estimated from the incoming signal, and distances corresponding to arriving signal paths are calculated based on ToA values [6].

In the measurement model, there are a number of (four in the experiments) UWB radar sensors, which constantly transmit signals, and the reflected signals from moving objects (in our case single/multiple person(s) in an indoor environment) are collected by each of these sensors as depicted in Fig. 1.

The output of each sensor is the range measurements related to moving objects. It is assumed that the locations of the sensors are known to the fusion center and each sensor sends its measurements to the fusion center. The state vector of a target at time $k$ is represented by $\mathbf{x}_{k}=\left[x_{k}, y_{k}, \dot{x}_{k}, \dot{y}_{k}\right]^{T}$, where $\left[x_{k}, y_{k}\right]$ is the position, $\left[\dot{x}_{k}, \dot{y}_{k}\right]$ is the velocity of the target and $T$ denotes transpose operation. The target dynamics is modeled by the nearly-constant-velocity model [30], [31]:

$$
\mathbf{x}_{k}=\mathbf{F} \mathbf{x}_{k-1}+\mathbf{v}_{k}
$$

where $k$ is the discrete time index, $\mathbf{F}$ is the state transition matrix given as

$$
\mathbf{F}=\left[\begin{array}{cc}
\mathbf{I}_{2} & \triangle \mathbf{I}_{2} \\
\mathbf{0}_{2} & \mathbf{I}_{2}
\end{array}\right]
$$

and $\mathbf{v}_{k} \sim \mathcal{N}(\mathbf{v} ; \mathbf{0}, \mathbf{Q})$ is the white Gaussian process noise, the covariance matrix of which is expressed as

$$
\mathbf{Q}=\sigma_{\mathbf{v}}^{2}\left[\begin{array}{cc}
\frac{\Delta^{3}}{3} \mathbf{I}_{2} & \frac{\Delta^{2}}{2} \mathbf{I}_{2} \\
\frac{\Delta^{2}}{2} \mathbf{I}_{2} & \triangle \mathbf{I}_{2}
\end{array}\right] .
$$

In (3) and (4), $\Delta$ denotes the sampling interval, $\sigma_{\mathbf{v}}$ is the standard deviation of the process noise, and $\mathbf{I}_{n}$, and $\mathbf{0}_{n}$ denote $n \times n$ identity and zero matrices, respectively. This model is also known as the white-noise-acceleration model due to the 
fact that the accelerations along $\mathrm{x}$-direction and $\mathrm{y}$-direction are modeled as white noise. Note that the word "nearly" used in name of the model, nearly-constant-velocity model, implies that these accelerations are actually small [30]. This model is simple, widely used, very appropriate for indoor tracking applications (i.e., in indoor areas, the motion of a target is constrained by the structured corridors and rooms), and effectively handles random maneuvers [32]-[34]. Moreover, by changing the value of the process noise standard deviation, $\sigma_{\mathbf{v}}$, one can adjust the acceleration noise intensity and can handle target maneuvers up to a certain level.

Range measurements are collected by each sensor in the area. The measured range value by the $i$-th sensor located at $\left[x_{i}, y_{i}\right]$ is given by

$$
h_{i}\left(\mathbf{x}_{k}\right)=\sqrt{\left(x_{k}-x_{i}\right)^{2}+\left(y_{k}-y_{i}\right)^{2}}+\varepsilon_{k, i}
$$

for $i=1, \ldots, N_{s}$, where $N_{s}$ is the number of sensors and $\varepsilon_{k, i}$ is measurement noise in sensor $i$, which is modeled as $\varepsilon_{k, i} \sim \mathcal{N}\left(\varepsilon ; 0, \sigma_{\varepsilon}^{2}\right)$.

The Jacobian of $h_{i}\left(\mathbf{x}_{k}\right), \mathbf{H}_{k, i}$, to be used in the filtering equations, is obtained as follows:

$$
\mathbf{H}_{k, i}=\left[\begin{array}{llll}
\frac{\partial h_{i}\left(\mathbf{x}_{k}\right)}{\partial x_{k}} & \frac{\partial h_{i}\left(\mathbf{x}_{k}\right)}{\partial y_{k}} & \frac{\partial h_{i}\left(\mathbf{x}_{k}\right)}{\partial \dot{x}_{k}} & \frac{\partial h_{i}\left(\mathbf{x}_{k}\right)}{\partial \dot{y}_{k}}
\end{array}\right]
$$

where the elements are expressed as

$$
\begin{aligned}
\frac{\partial h_{i}\left(\mathbf{x}_{k}\right)}{\partial x_{k}} & =\frac{x_{k}-x_{i}}{\sqrt{\left(x_{k}-x_{i}\right)^{2}+\left(y_{k}-y_{i}\right)^{2}}} \\
\frac{\partial h_{i}\left(\mathbf{x}_{k}\right)}{\partial y_{k}} & =\frac{y_{k}-y_{i}}{\sqrt{\left(x_{k}-x_{i}\right)^{2}+\left(y_{k}-y_{i}\right)^{2}}} \\
\frac{\partial h_{i}\left(\mathbf{x}_{k}\right)}{\partial \dot{x}_{k}} & =0 \\
\frac{\partial h_{i}\left(\mathbf{y}_{k}\right)}{\partial \dot{y}_{k}} & =0 .
\end{aligned}
$$

\section{RANDOM Finite SETS (RFS) BASED FILTERING}

The RFS framework for multiple target tracking proposed by Mahler combines the problems of combinatorial data association, detection, classification and target tracking within a unified compact Bayesian paradigm [19]. In the following subsections, the basic RFS notation, multiple target generalization of the Bayes filter and its first order approximation PHD filter are described.

\section{A. RFS Formulation}

The RFS approach treats the collection of the individual targets and individual measurements as a set-valued state and set-valued measurement, respectively, as

$$
\begin{aligned}
X_{k} & =\left\{\mathbf{x}_{k, 1}, \ldots, \mathbf{x}_{k, M(k)}\right\} \quad \in \mathcal{F}(\mathcal{X}) \\
Z_{k} & =\left\{z_{k, 1}, \ldots, z_{k, N(k)}\right\} \quad \in \mathcal{F}(\mathcal{Z})
\end{aligned}
$$

where $M(k)$ is the number of targets at time $k, N(k)$ is the number of measurements at time $k, \mathcal{F}(\mathcal{X})$ and $\mathcal{F}(\mathcal{Z})$ are the set of all possible finite subsets of state space $\mathcal{X}$ and measurement space $\mathcal{Z}$, respectively. An RFS model for the time evolution of a multi-target state $X_{k-1}$ at time $k-1$ to the multi-target state $X_{k}$ at time $k$ is defined as

$$
X_{k}=\left[\bigcup_{\zeta \in X_{k-1}} S_{k \mid k-1}(\zeta)\right] \cup \Gamma_{k}
$$

where $S_{k \mid k-1}(\zeta)$ is the RFS of surviving targets from previous state $\zeta$ at time $k$ and $\Gamma_{k}$ is the RFS of spontaneous target births at time $k$. The RFS measurement model for a multi-target state $X_{k}$ at time $k$ can be written as

$$
Z_{k}=K_{k} \cup\left[\bigcup_{\mathbf{x} \in X_{k}} \Theta_{k}(\mathbf{x})\right]
$$

where $K_{k}$ is the RFS of clutter or false measurements, and $\Theta_{k}(\mathbf{x})$ is the RFS of multi-target state originated measurements, which can take values of either $z_{k}$ if target is detected, or $\emptyset$ if no target is detected.

\section{B. Multi-Target Filtering}

Having very briefly summarized some key points of the RFS framework, we can define the RFS based multi-target Bayes filter. The optimal multi-target Bayes filter propagates the multi-target posterior density $p_{k}\left(\cdot \mid Z_{1: k}\right)$ conditioned on the sets of measurements up to time $k, Z_{1: k}$, in time with the following recursion:

$$
\begin{aligned}
p_{k \mid k-1}\left(X_{k} \mid Z_{1: k-1}\right) & =\int f_{k \mid k-1}\left(X_{k} \mid X\right) p_{k-1}\left(X \mid Z_{1: k-1}\right) \delta X \\
p_{k}\left(X_{k} \mid Z_{1: k}\right) & =\frac{g_{k}\left(Z_{k} \mid X_{k}\right) p_{k \mid k-1}\left(X_{k} \mid Z_{1: k-1}\right)}{\int g_{k}\left(Z_{k} \mid X\right) p_{k \mid k-1}\left(X \mid Z_{1: k-1}\right) \delta X}
\end{aligned}
$$

where $f_{k \mid k-1}$ is the multi-target transition density, $g_{k}\left(Z_{k} \mid X_{k}\right)$ is the multi-target likelihood and the integrals are set integrals as defined in [19]. The multi-target Bayes recursion involves multiple integrals and the complexity of computing it grows exponentially with the number of targets. Therefore, it is not practical for scenarios where there exist more than a few targets.

\section{The Probability Hypothesis Density (PHD) Filter}

To alleviate the computational burden in calculating the optimal filter given above, the PHD filter was proposed as a practical suboptimal alternative [19]. The PHD filter propagates the first-order statistical moment of the posterior multi-target state, instead of propagating the multi-target posterior density. Let the intensities associated with the multi-target posterior density $p_{k}$ and the multitarget predicted density $p_{k \mid k-1}$ in the optimal multi-target Bayes recursion be represented by $v_{k}$ and $v_{k \mid k-1}$, respectively. Then, the PHD recursion is defined as

$$
\begin{aligned}
v_{k \mid k-1}(\mathbf{x})= & \int p_{s} f_{k \mid k-1}(\mathbf{x} \mid \zeta) v_{k-1}(\zeta) d \zeta+\gamma_{k}(\mathbf{x}) \\
v_{k}(\mathbf{x})= & \left(1-p_{D}\right) v_{k \mid k-1}(\mathbf{x}) \\
& +\sum_{z \in Z_{k}} \frac{p_{D} g_{k}(z \mid \mathbf{x}) v_{k \mid k-1}(\mathbf{x})}{\kappa_{k}(z)+\int p_{D} g_{k}(z \mid \boldsymbol{\xi}) v_{k \mid k-1}(\boldsymbol{\xi}) d \boldsymbol{\xi}}
\end{aligned}
$$


where $p_{s}$ is the probability of target survival, $\gamma_{k}(\mathbf{x})$ is the intensity of spontaneous birth RFS at time $k, p_{D}$ is the probability of target detection and $\kappa_{k}(z)$ is the intensity of clutter RFS at time $k$.

As mentioned before, PHD filters can be implemented either by using GM [35] or SMC [36]-[38] based methods. In the next section, we describe the main steps of the GM implementation.

\section{The Gaussian Mixture PHD (GM-PHD) Filter}

The closed-form solution to the PHD filter, called the Gaussian mixture PHD (GM-PHD), is originally derived for linear Gaussian multi-target models, since it makes use of the Kalman filter to propagate the mean and covariance of each Gaussian [39]. In addition, to accommodate nonlinear dynamics and measurement models, several different nonlinear extensions of the GM-PHD are also proposed in the literature [40], [41]. These nonlinear extensions of the GM-PHD filter have successfully been used in many different applications, in which nonlinear target dynamics and measurement models are employed [42]- [48]. In this work, in order to accommodate nonlinear Gaussian models, an adaptation of the GM-PHD filter (called as EK-PHD) is employed based on the idea of extended Kalman filter (EKF), where the local linearization of the nonlinear measurement function $h(\mathbf{x})$ (i.e., $\mathbf{H}_{k}$ defined in (6)) is used [39]. We use this adaptation approach to handle the nonlinearities in the measurement model in (5).

There are several assumptions used in the GM-PHD recursions. The first one is that each target follows a linear Gaussian dynamical and measurement model:

$$
\begin{aligned}
f_{k \mid k-1}(\mathbf{x} \mid \zeta) & =\mathcal{N}\left(\mathbf{x} ; \mathbf{F} \zeta, \mathbf{Q}_{k-1}\right) \\
g_{k}(z \mid \mathbf{x}) & =\mathcal{N}\left(z ; \mathbf{H}_{k} \mathbf{x}, \sigma_{\varepsilon}^{2}\right) .
\end{aligned}
$$

Secondly, the detection and survival probabilities are state and time independent; that is, $p_{D, k}(\mathbf{x})=p_{D}$ and $p_{S}(\mathbf{x})=p_{S}$. Lastly, the intensity of the birth RFSs is Gaussian mixture of the form

$$
\gamma_{k}(x)=\sum_{i=1}^{J_{\gamma, k}} w_{\gamma, k}^{(i)} \mathcal{N}\left(\mathbf{x} ; \mathbf{m}_{\gamma, k}^{(i)}, \mathbf{P}_{\gamma, k}^{(i)}\right)
$$

where $J_{\gamma, k}, w_{\gamma, k}^{(i)}, \mathbf{m}_{\gamma, k}^{(i)}$ and $\mathbf{P}_{\gamma, k}^{(i)}$ are the given model parameters that determine the birth intensity. The posterior intensity at time $k-1$ can be written as a sum of Gaussian components with different weights, means and covariances as

$$
v_{k-1}(\mathbf{x})=\sum_{i=1}^{J_{k-1}} w_{k-1}^{(i)} \mathcal{N}\left(\mathbf{x} ; \mathbf{m}_{k-1}^{(i)}, \mathbf{P}_{k-1}^{(i)}\right)
$$

and an identifying label $\ell_{k-1}^{i}$ is assigned to each created Gaussian component. A label table, $\mathcal{L}_{k-1}$, is formed as

$$
\mathcal{L}_{k-1}=\left\{\ell_{k-1}^{(1)}, \ldots, \ell_{k-1}^{\left(J_{k-1}\right)}\right\} .
$$

At time $k$, the predicted intensity is also a Gaussian mixture:

$$
v_{k \mid k-1}(\mathbf{x})=v_{S, k \mid k-1}(\mathbf{x})+\gamma_{k}(\mathbf{x})
$$

where

$$
\begin{aligned}
v_{S, k \mid k-1}(\mathbf{x}) & =p_{S} \sum_{j=1}^{J_{k-1}} w_{k-1}^{(j)} \mathcal{N}\left(\mathbf{x} ; \mathbf{m}_{S, k \mid k-1}^{(j)}, \mathbf{P}_{S, k \mid k-1}^{(j)}\right) \\
\mathbf{m}_{S, k \mid k-1}^{(j)} & =\mathbf{F} \mathbf{m}_{k-1}^{(j)} \\
\mathbf{P}_{S, k \mid k-1}^{(j)} & =\mathbf{Q}_{k-1}+\mathbf{F} \mathbf{P}_{k-1}^{(j)} \mathbf{F}^{T}
\end{aligned}
$$

Each birth component is assigned a new label and concatenated with the previous time labels, i.e.,

$$
\mathcal{L}_{k \mid k-1}=\mathcal{L}_{k-1} \cup \mathcal{L}_{\gamma, k-1} .
$$

The posterior intensity at time $k$ is also a Gaussian mixture and can be written as

$$
v_{k}(\mathbf{x})=\left(1-p_{D, k}\right) v_{k \mid k-1}(\mathbf{x})+\sum_{z \in Z_{k}} v_{D, k}(\mathbf{x} ; z)
$$

where

$$
\begin{aligned}
v_{D, k}(\mathbf{x} ; z) & =\sum_{j=1}^{J_{k \mid k-1}} w_{k}^{(j)}(z) \mathcal{N}\left(\mathbf{x} ; \mathbf{m}_{k \mid k}^{(j)}(z), \mathbf{P}_{k \mid k}^{(j)}\right) \\
w_{k}^{(j)}(z) & =\frac{p_{D} w_{k \mid k-1}^{(j)} q_{k}^{(j)}(z)}{\kappa_{k}(z)+p_{D} \sum_{l=1}^{J_{k \mid k-1}} w_{k \mid k-1}^{(l)} q_{k}^{l}(z)} \\
q_{k}^{j}(z) & =\mathcal{N}\left(z ; \mathbf{H}_{k} \mathbf{m}_{k \mid k-1}^{(j)}, \sigma_{\varepsilon}^{2}+\mathbf{H}_{k} \mathbf{P}_{k \mid k-1}^{(j)} \mathbf{H}_{k}^{T}\right) \\
\mathbf{m}_{k \mid k}^{(j)}(z) & =\mathbf{m}_{k \mid k-1}^{(j)}+\mathbf{K}_{k}^{(j)}\left(z-\mathbf{H}_{k} \mathbf{m}_{k \mid k-1}^{(j)}\right) \\
P_{k \mid k}^{(j)} & =\left[\mathbf{I}-\mathbf{K}_{k}^{(j)} \mathbf{H}_{k}\right] \mathbf{P}_{k \mid k-1}^{(j)} \\
\mathbf{K}_{k}^{(j)} & =\mathbf{P}_{k \mid k-1}^{(j)} \mathbf{H}_{k}^{T}\left(\mathbf{H}_{k} \mathbf{P}_{k \mid k-1}^{(j)} \mathbf{H}_{k}^{T}+\sigma_{\varepsilon}^{2}\right)^{-1}
\end{aligned}
$$

There will be $\left|Z_{k}\right|+1$ Gaussian components for each predicted term, where $|\cdot|$ is the cardinality of a set. Then, the identifying label at time $k$ is

$$
\mathcal{L}_{k}=\mathcal{L}_{k \mid k-1}^{v_{k \mid k-1}} \cup \mathcal{L}_{k \mid k-1}^{z_{1}} \cup \ldots \cup \mathcal{L}_{k \mid k-1}^{z_{\mid} Z_{k} \mid} .
$$

As time progresses, the number of Gaussian components increases and computational problems occur. To alleviate this problem, a simple pruning and merging can be used to decrease the number of Gaussian components propagated [35]. Firstly, the weights below a predefined threshold are eliminated. Then, the closely spaced Gaussian components are merged into a single Gaussian component. Starting with the strongest weighted component, $w_{k}^{j}$, components are merged in a set $W_{k}^{(j)}$ as

$$
W_{k}^{(j)}:=\left\{i:\left(\mathbf{m}_{k}^{(i)}-\mathbf{m}_{k}^{(j)}\right)^{T}\left(\mathbf{P}_{k}^{(i)}\right)^{-1}\left(\mathbf{m}_{k}^{(i)}-\mathbf{m}_{k}^{(j)}\right) \leq \rho\right\}
$$

and the resulting merged component parameters are

$$
\begin{aligned}
\tilde{w}_{k}^{(l)} & =\sum_{i \in W} w_{k}^{(i)} \\
\tilde{\mathbf{m}}_{k}^{(l)} & =\frac{1}{\tilde{w}_{k}^{(l)}} \sum_{i \in W} w_{k}^{(i)} \mathbf{x}_{k}^{(i)} \\
\tilde{\mathbf{P}}_{k}^{(l)} & =\frac{1}{\tilde{w}_{k}^{(l)}} \sum_{i \in W} w_{k}^{(i)}\left(\mathbf{P}_{k}^{(i)}+\left(\tilde{\mathbf{m}}_{k}^{(l)}-\mathbf{m}_{k}^{(i)}\right)\left(\tilde{\mathbf{m}}_{k}^{(l)}-\mathbf{m}_{k}^{(i)}\right)^{T}\right)
\end{aligned}
$$




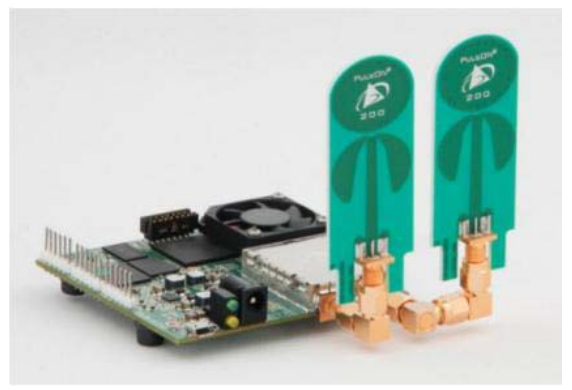

Fig. 2. P410 MRM with attached broadspec antennas [29].

In order to extract multi-target states, the means of the Gaussian components that have weights greater than some predefined threshold, are selected:

$$
\hat{\mathcal{L}}_{k}=\left\{\mathcal{L}_{k}^{(i)}: w_{k}^{(i)}>\rho\right\}
$$

and the estimated target states set is expressed as

$$
\hat{X}_{k}=\left\{\left(\mathbf{m}_{k}^{(i)}, \mathbf{P}_{k}^{(i)}\right): \mathcal{L}_{k}^{(i)} \in \hat{\mathcal{L}}_{k}\right\} \text {. }
$$

\section{UWB RADAR SENSORS}

In the experiments, four UWB radar sensors named P410 Monostatic Radar Module (MRM) are used [29]. P410 MRM, shown in Fig. 2, is a small and affordable monostatic radar platform that provides more than $2 \mathrm{GHz}$ of RF bandwidth at a center frequency of $4.3 \mathrm{GHz}$. Each radar sensor is equipped with an UWB transmitter and an UWB receiver. The radar sensors use different code channels in order to prevent interference among the sensors. In addition, for reducing the effects of severe multipaths at the receiver, there is an environment scanning phase for a $30 \mathrm{~ns}$ duration, which is used as a reference for determining signals reflected from nonstationary objects. The UWB pulses are sent from the radar sensors at every 0.1 second by the transmitter (TX) antenna and all reflected signals are collected by the receiver (RX) antenna. P410 MRM UWB sensors provide four types of information; raw signal, bandpass signal, motion filtered signal, and detection list. In some cases, the motion filtered data and detection lists may not be sufficient to detect the targets accurately since there can be many unnecessary measurements (due to the very high resolution of UWB signals) that are originated from the reflections from other equipments or objects in the environment. Therefore, we use the bandpass data (see Fig. 3 for an example) in our algorithm in order to eliminate clutter, and then obtain the motion filtered data, as explained in the next section. Fig. 4 presents an example of motion filtered data when a person is present in the environment.

\section{Vi. Proposed Detection/Tracking Algorithm}

In order to perform accurate detection and tracking of multiple persons via UWB radar sensors, the following algorithm is proposed. The input to the algorithm is the bandpass signal sets from the UWB radar sensors. Fig. 3 illustrates an example of bandpass signal at an arbitrary time stamp. After getting the bandpass signal sets from the radar sensors, the start time $\left(t_{s}\right)$

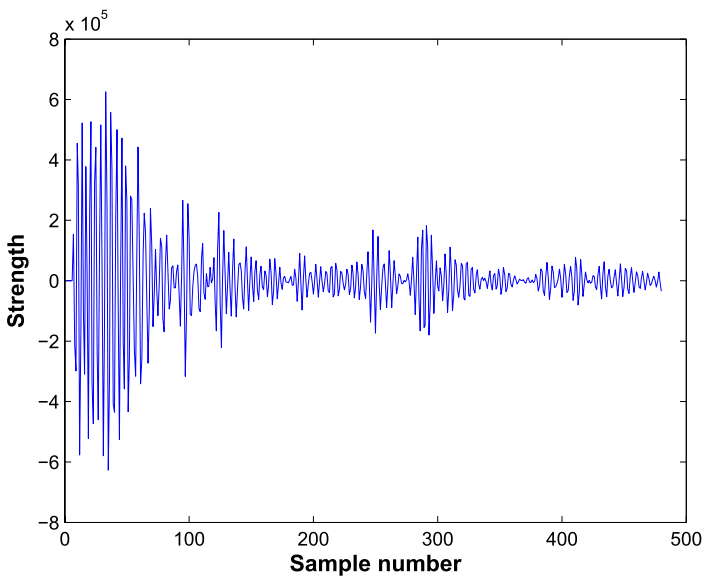

Fig. 3. Bandpass signal.

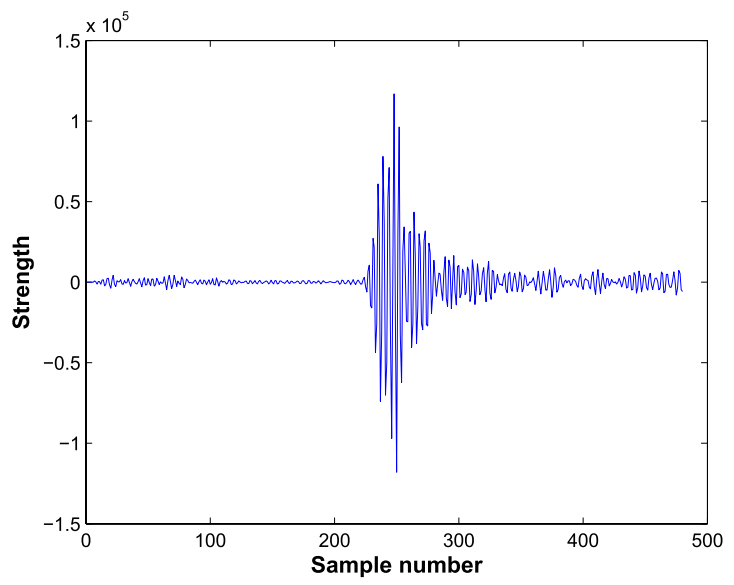

Fig. 4. Motion filtered data.

and stop time $\left(t_{f}\right)$ of the experiment are calculated. In total, $N_{d}$ sets of measurements are obtained from the sensors, where $N_{d}$ is given by

$$
N_{d}=\frac{t_{f}-t_{s}}{T_{s}}
$$

with $T_{S}$ representing the sampling period of the signal set, which is equal for all sensors. The bandpass signal set is filtered by a motion filter in order to mitigate the effects of the signals coming from stationary objects in the environment. The following motion filtering method is employed:

$$
\begin{aligned}
m_{k}^{i}[n]= & h[1] r_{k}^{i}[n]+h[2] r_{k}^{i}[n-1] \\
& +h[3] r_{k}^{i}[n-2]+h[4] r_{k}^{i}[n-3]
\end{aligned}
$$

for $i \in\{1, \ldots, 4\}$ and $k \in\left\{1, \ldots, N_{d}\right\}$, where $r_{k}^{i}[n]$ represents the bandpass signal of the $i$ th radar sensor for the $k$ th measurement set, $h[n]$ denotes the coefficients of the motion filter with values $[1-0.6-0.3-0.1]$, and $N_{d}$ is the number of measurement sets as defined in (43). In other words, for each measurement set and for each sensor, the motion filter in (44) is applied to the bandpass signal, and the motion filtered signal $m_{k}^{i}[n]$ is generated. The peaks of the motion filtered signal correspond to possible target distances as can be observed from Fig. 4 


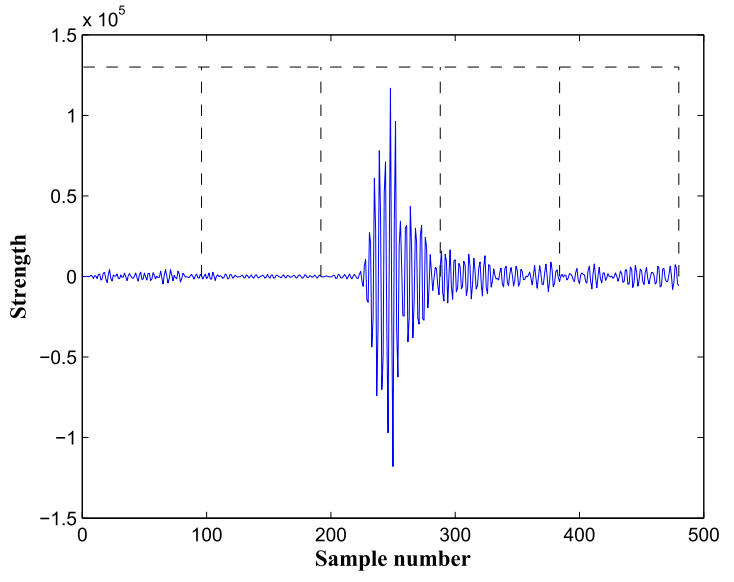

Fig. 5. Blocks of motion filtered signal.

Next, each motion filtered signal is divided into $\left(N_{b}\right)$ blocks as follows:

$$
m_{k, j}^{i}[n]=m_{k}^{i}[n+(j-1) K], \quad n=1, \ldots, K
$$

for $j \in\left\{1, \ldots, N_{b}\right\}$, where $K$ is the number of samples in each block, which is considered as constant. ${ }^{1}$ An example illustration is presented in Fig. 5, where $N_{b}=5$. The aim of dividing the motion filtered signal into blocks is to increase both the efficiency and the speed of the proposed algorithm, which can be justified as follows. Due to the very high time resolution of UWB signals, there exist many peaks in the motion filtered signal, most of which are originated from the same targets (that is, each moving object/person results in many peaks in the motion filtered signal). In order to determine the number of targets accurately (hence, to track them efficiently), only a few significant motion filter peaks should be considered, which is facilitated by the proposed block operation in (45) (and the energy thresholding technique explained below). This operation also increases the speed of the algorithm since a smaller set of measurements are input to the tracking part of the algorithm. The number of blocks, $N_{b}$, is an important parameter, which should be selected according to the number of expected targets in the environment. As the number of targets increases, $N_{b}$ should be set to a larger number.

Once the motion filtered signal is divided into blocks, the average strength of each block is calculated as follows:

$$
E_{k, j}^{i} \triangleq \frac{1}{K} \sum_{n=1}^{K}\left|m_{k, j}^{i}[n]\right|
$$

for $j \in\left\{1, \ldots, N_{b}\right\}, i \in\{1, \ldots, 4\}$, and $k \in\left\{1, \ldots, N_{d}\right\}$. Then, these values are compared to a threshold $\tau_{i}$ for each sensor in order to eliminate the blocks that do not contain signals from the targets. In other words, if the average strength of a block is below the threshold, then that block is not considered in the next steps. This process both reduces the

\footnotetext{
${ }^{1}$ For simplicity of notation, the size of signal $m_{k}^{i}[n]$ is assumed to be an integer multiple of $K$. Extensions in the absence of this assumption are straightforward.
}

TABLE I

PROPOSED ALGORITHM

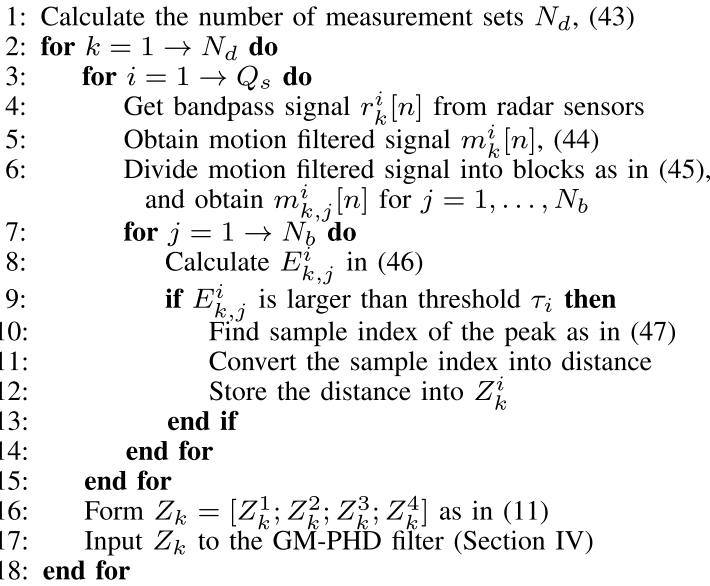

computational complexity and number of detections. If the average strength of a block is larger than the threshold, then the sample index of the strongest motion filter output in that block is converted into distance (meters) and stored into the measurement vector $Z_{k}^{i}$. Mathematically, for $j \in\left\{1, \ldots, N_{b}\right\}$, if $E_{k, j}^{i}>\tau_{i}$, then the sample index

$$
\underset{n \in\{1, \ldots, K\}}{\arg \max }\left|m_{k, j}^{i}[n]\right|
$$

is converted into distance and saved into $Z_{k}^{i}$. Therefore, $Z_{k}^{i}$ is a vector with $G_{k}^{i}$ measurements, where $G_{k}^{i} \in\left\{0,1, \ldots, N_{b}\right\}$ is the number of blocks that satisfy $E_{k, j}^{i}>\tau_{i}{ }^{2}$ Measurements from all four sensors are collected into measurement set $Z_{k}$ as in (11); that is, $Z_{k}=\left[Z_{k}^{1} ; Z_{k}^{2} ; Z_{k}^{3} ; Z_{k}^{4}\right]$. Then, $Z_{k}$ is input to the GM-PHD filter described in Section IV, and tracking is performed. The proposed detection and tracking algorithm is summarized in Table I.

\section{EXPERIMENTAL RESULTS}

Experimental results for single and multiple person are presented in this section. The experiments are performed in an office room in the Department of Electrical and Electronics Engineering at Bilkent University. There are many equipments/objects which can generate multipaths in the office environment as seen in Fig 6. In the experiments, four P410 MRMs are used. In order to reduce the number of detections and the computational complexity of the algorithm, the number of blocks is set to six in the algorithm; that is, $N_{b}=6$ (see (45)). The threshold $\tau_{i}$ in Section VI is set to 12000 (in units of P410 MRM outputs) in order to determine and eliminate noise only blocks, and the sampling period $T_{s}$ is taken as 0.1 second.

The standard deviation of the process noise is taken as $\sigma_{\mathbf{v}}=2 \mathrm{~m} / \mathrm{s}^{2}$ and the standard deviation of the measurement noise is taken as $\sigma_{\varepsilon}=0.2 \mathrm{~m}$. The spontaneous birth intensities are described in the center of the tracking area since the birth locations are assumed as unknown. Hence, our method

\footnotetext{
${ }^{2}$ If the strengths of all the blocks are below $\tau_{i}$, then $Z_{k}^{i}$ becomes an empty vector, $Z_{k}^{i}=\emptyset$.
} 


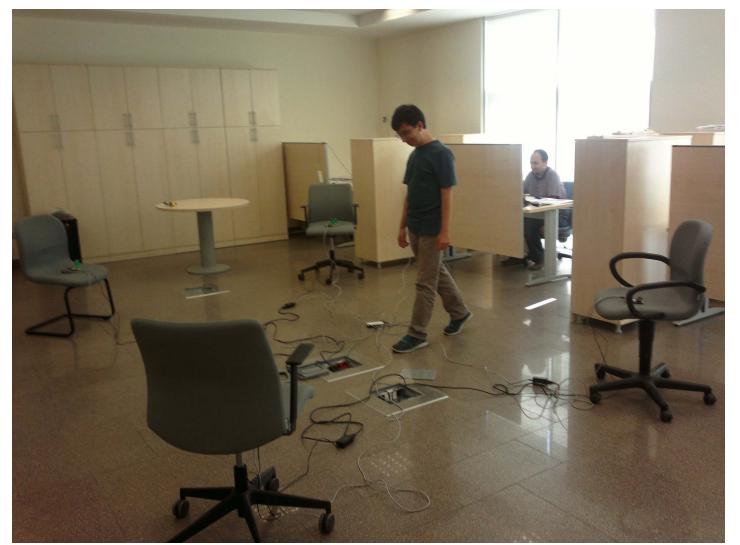

Fig. 6. An illustration of the office environment. Four radar sensors are placed on the chairs.



Fig. 7. Scenario-1 for single-person tracking. Blue solid line and red circles represent the ground truth and filter estimates, respectively. Black squares are for UWB radar sensors.

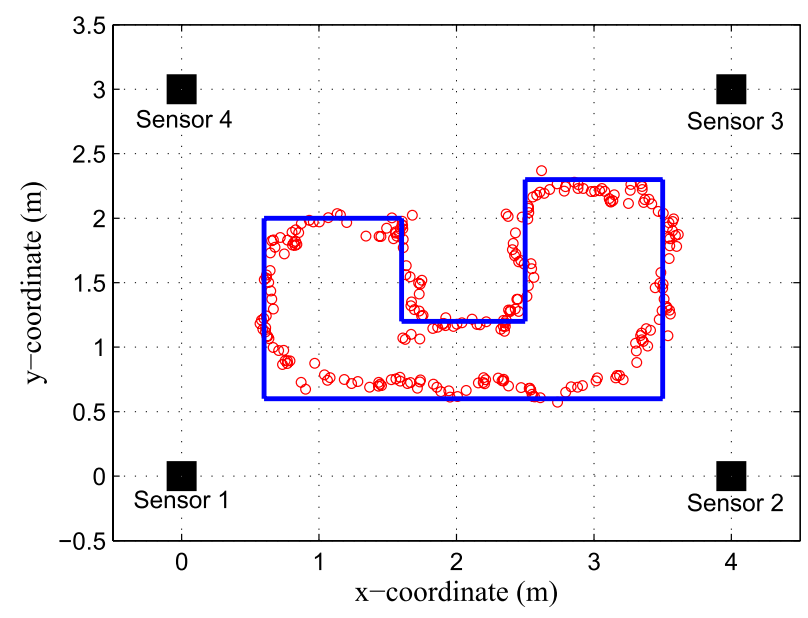

Fig. 8. Scenario-2 for single-person tracking. Blue solid line and red circles represent the ground truth and filter estimates, respectively. Black squares are for UWB radar sensors.

can be applied to any scenario and we do not need to describe the birth intensities even though the tracking is lost. In the algorithm, the initial weights which are described in Section IV as in (21) are taken as $w_{i}=0.1$. In pruning parameters, the truncation threshold for the weights is chosen

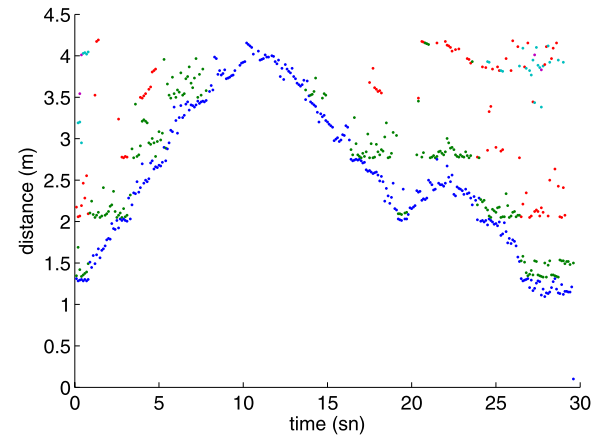

(a)



(b)



(c)

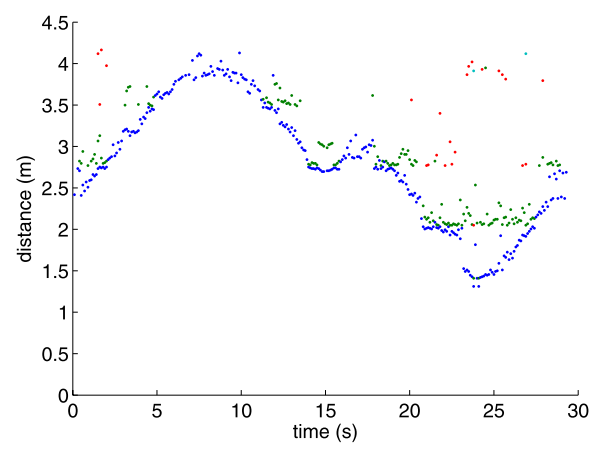

(d)

Fig. 9. Range measurements of sensors in the single-person tracking scenario (Scenario-2). (a) Sensor 1, (b) Sensor 2, (c) Sensor 3, (d) Sensor 4.

as $\rho=10^{-6}$ and the maximum allowable number of Gaussian terms is taken as 20. In our scenario, the tracking scenario is not very complicated; hence, this number is set to 20 in order to have a faster result. However, in complex cases, this component number can be increased. In addition, clutter is 


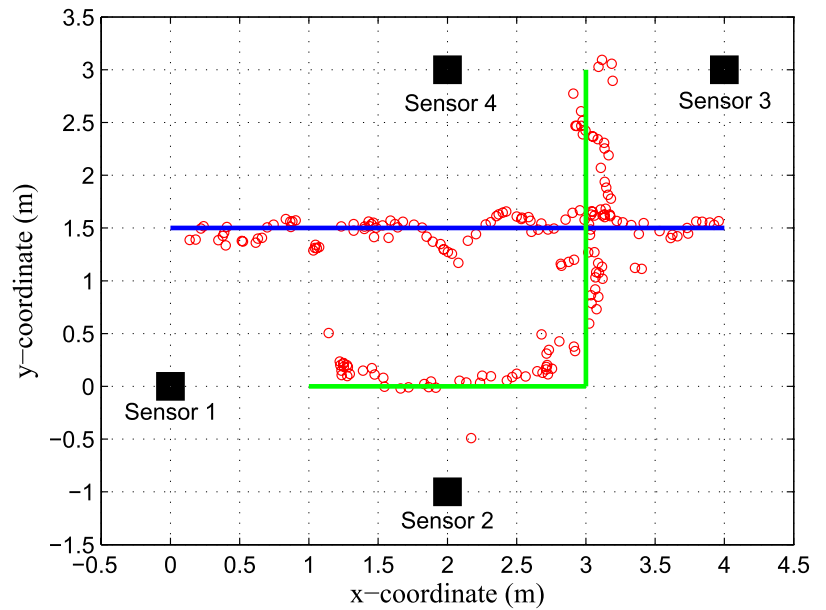

Fig. 10. Scenario-1 for multiple person tracking. Green and blue solid lines denote the ground truth of the first and second person, respectively. Black squares are for UWB radar sensors.

modeled according to the highest and smallest measurements. The detected measurements are immersed in clutter that can be modelled as Poisson RFS $K_{k}$ with intensity

$$
\kappa_{k}(z)=\lambda_{c} V u(z)
$$

where $u(\cdot)$ is the uniform density over the surveillance region, $V=12 \mathrm{~m}^{2}$ is the "volume" of the surveillance region, and $\lambda_{c}=0.417 \mathrm{~m}^{-2}$ is the average number of clutter returns per unit volume (i.e., 5 clutter returns over the surveillance region).

Our computer has $8 \mathrm{~GB}$ RAM and its processor is $3.40 \mathrm{GHz}$ Intel(R) Core(TM) i7. The proposed approach runs at real-time and one iteration of the experiment takes approximately $1 \mathrm{~s}$ on the average.

\section{A. Single-Person Tracking Results}

In the first set of experiments, we consider the tracking of one person and study two different scenarios. In the first scenario (Scenario-1), the person (target) starts from position $(0,2.5) \mathrm{m}$. and walks in a straight line until $(2.8,2.5) \mathrm{m}$. Then, he turns right and walks until $(2.8,0.5) \mathrm{m}$. After that, he again turns right and goes until $(0,0.5) \mathrm{m}$. The person walks with a speed of around $0.4 \mathrm{~m} / \mathrm{s}$, and the experiment takes about 19 seconds. The results are shown in Fig. 7, where the blue line is the ground truth of the target path, and the red circles are the estimates of the proposed algorithm. The width of the person is about $0.5 \mathrm{~m}$ and reflections from different parts of the body, such as legs, arms, and the center of the body, are received at different positions due to the high resolution of UWB signals. Therefore, the blue line is in fact the approximate ground truth for the path of the person. Therefore, the red circles slightly digress from the blue line as expected. (The receiver noise and multipath can also contribute to position estimation errors.) The differences between the blue line and the red circles are always smaller than $0.25 \mathrm{~m}$ in this scenario, which indicates that the positions of the person can be estimated accurately by the proposed algorithm in this case.

The second scenario (Scenario-2) for the single target case involves a more challenging target path with target maneuvers in a small area. In this scenario, the target starts to move from

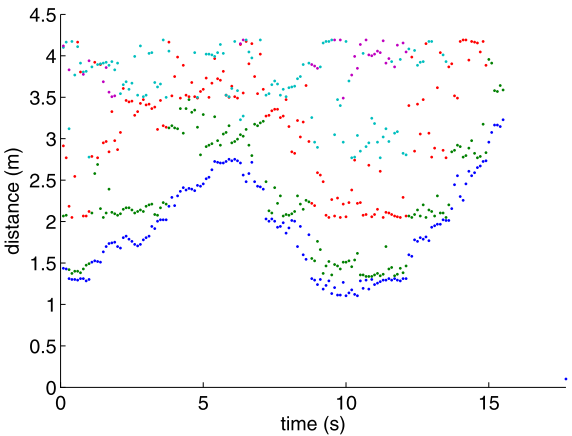

(a)

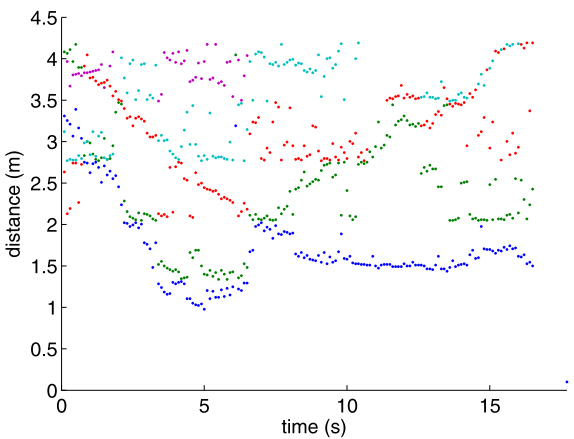

(b)



(c)

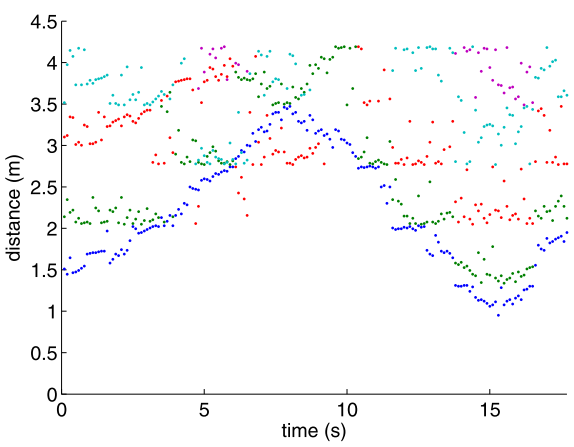

(d)

Fig. 11. Range measurements of sensors in the multi-person tracking scenario (Scenario-1). (a) Sensor 1, (b) Sensor 2, (c) Sensor 3, (d) Sensor 4.

position $(0.6,0.7) \mathrm{m}$. and comes back to the same position after following the blue path in Fig. 8. The duration of the experiment is approximately $29 \mathrm{~s}$. Similar to the previous experiment, the red circles in Fig. 8 are very close to the real path and the algorithm performs very well for this difficult scenario. 


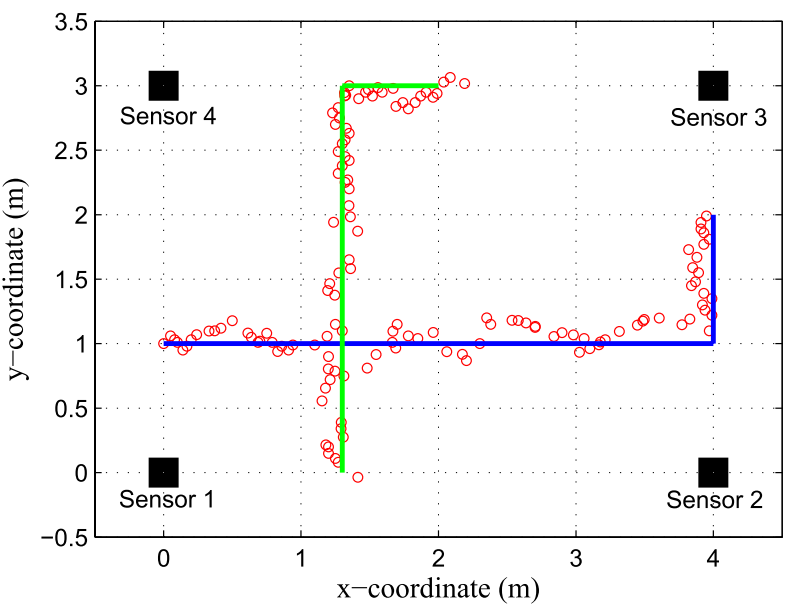

Fig. 12. Scenario-2 for multiple person tracking. Green and blue solid lines denote the ground truth of the first and second person, respectively. Black squares are for UWB radar sensors.

In Fig. 9, the detection data obtained from the four UWB radar sensors are illustrated for the scenario in Fig. 8. As discussed in Section VI, the indices of the strongest samples are calculated for the blocks of motion filtered data that have an average value larger than the threshold. Therefore, in the figures, the number of points at each time instant indicates the number of blocks the average strength of which are above the threshold (equivalently, the number of elements in $Z_{k}^{i}$ for a given time index $k$ and sensor index $i$; see Section VI). The points in the figures are color coded in such a way that the colors blue, green, red, cyan, and magenta are employed in the order of increasing distances from the sensors; that is, the blue and purple points are used for the detection points that are closest to and furthest away from the given sensor, respectively. It is observed from Fig. 9 that there are many non-target detections due to the high time resolution of UWB signals. However, the proposed approach can successfully eliminate the clutter and provide accurate tracking results, as shown Fig. 8.

\section{B. Multi-Person Tracking Results}

Next, we consider cases in which multiple persons are walking. First, we choose to perform controlled experiments for two persons. The first person starts from the position $(3,3) \mathrm{m}$. and the other person starts from the position $(0,1.5) \mathrm{m}$. The first person walks until $(3,0) \mathrm{m}$. and turns right. Then, he walks until $(1,0) \mathrm{m}$. His velocity is about $0.3 \mathrm{~m} / \mathrm{s}$. The second person walks in a straight line and its velocity is about $0.25 \mathrm{~m} / \mathrm{s}$. The experiment takes about 16 seconds. The ground truths are shown in Fig. 10 with the blue and green lines. The red circles represent the estimation results and they are commonly in the range of the human body. Therefore, for the multiple person case, the algorithm performs well in this scenario. There are some differences between the single and multiple person tracking scenarios. For instance, the sensor measurements are more complicated in the multiple person case, which can be observed by comparing the sensor data in Fig. 11 with that in Fig. 9. In particular, when there are a larger number of detection points (represented by different colors)

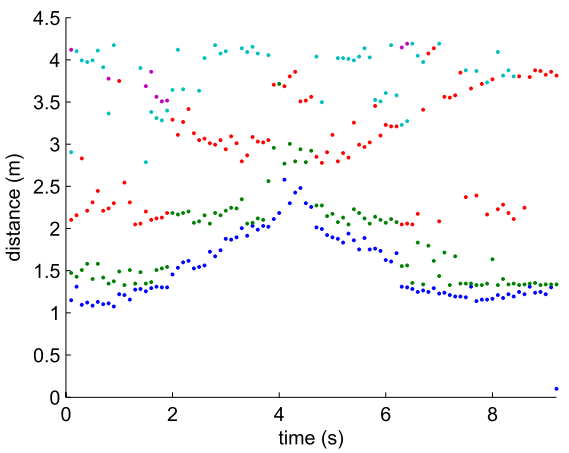

(a)

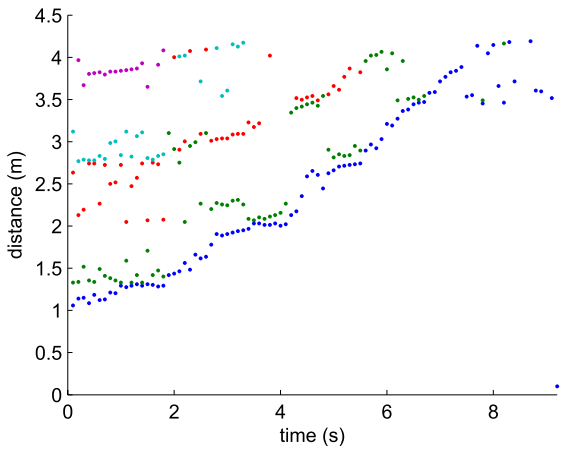

(b)

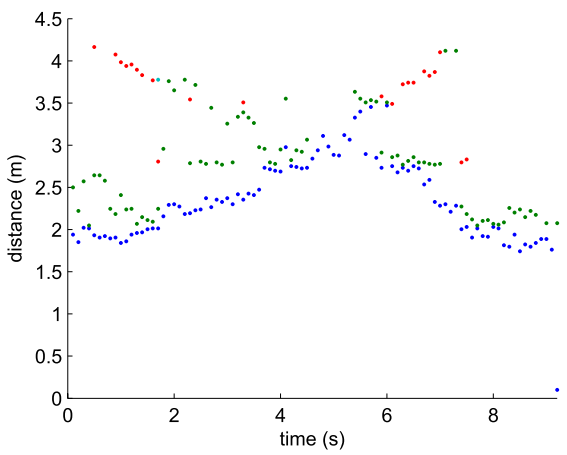

(c)

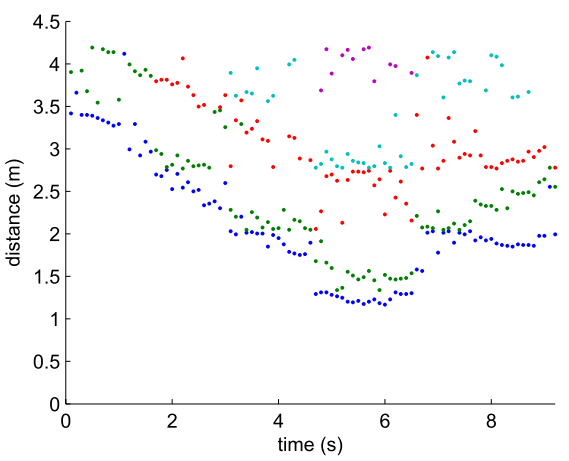

(d)

Fig. 13. Range measurements of sensors in the multi-person tracking scenario (Scenario-2). (a) Sensor 1, (b) Sensor 2, (c) Sensor 3, (d) Sensor 4.

for a time instant, target originated detections and clutters are observed more frequently, which makes person tracking more challenging.

One of the difficult tracking scenarios is the one shown in Fig. 12 since there is an occlusion problem as the sensors may not detect the locations of persons when they are 
in the same line. In general, the occlusion problem makes the situation quite complicated. In this experiment, there are two persons with the first one starting from $(1.25,0) \mathrm{m}$. and the second one from $(4,2) \mathrm{m}$. The first person goes to $(1.25,3) \mathrm{m}$. and then continues to walk until $(2,3) \mathrm{m}$. while the second person goes to $(4,1) \mathrm{m}$. and then finishes his walk until $(0,1) \mathrm{m}$. The red circles are the location estimates for the persons. In this case, some points cannot be detected. However, most of the time, the targets (persons) can be tracked with high accuracy by the proposed algorithm.

The detection data of the sensors for the scenario in Fig. 12 are shown in Fig. 13. Processing the data is quite difficult in this case since there are many clutters; in addition, when the targets are closer to each other, they cannot be detected separately because of the occlusion problem. However, the proposed algorithm still provides accurate tracking in this challenging scenario.

\section{CONCLUdING REMARKS}

In this study, multi-person tracking has been performed in an indoor environment via UWB radar sensors. A detection algorithm has been proposed and GM-PHD filtering has been employed for accurate target tracking. A development kit from TimeDomain has been used to collect data in an office environment. Based on the data collection campaigns, the performance of the proposed algorithm has been evaluated and it has been shown that it can track single and multiple targets accurately in various scenarios.

\section{REFERENCES}

[1] W. Dargie and C. Poellabauer, Fundamentals of Wireless Sensor Networks: Theory and Practice. New York, NY, USA: Wiley, 2010.

[2] K. Sohraby, D. Minoli, and T. Znati, Wireless Sensor Networks: Technology, Protocols, and Applications. New York, NY, USA: Wiley, 2007.

[3] J.-Y. Lee and R. Scholtz, "Ranging in a dense multipath environment using an UWB radio link," IEEE J. Sel. Areas Commun., vol. 20, no. 9, pp. 1677-1683, Dec. 2002.

[4] S. Gezici, Z. Sahinoglu, A. F. Molisch, H. Kobayashi, and H. V. Poor, "Two-step time of arrival estimation for pulse based ultra-wideband systems," EURASIP J. Adv. Signal Process., vol. 2008, May 2008, Art. ID 529134

[5] M. Chiani and A. Giorgetti, "Coexistence between UWB and narrowband wireless communication systems," Proc. IEEE, vol. 97, no. 2, pp. 231-254, Feb. 2009.

[6] Z. Sahinoglu, S. Gezici, and I. Guvenc, Ultra-Wideband Positioning Systems: Theoretical Limits, Ranging Algorithms, and Protocols. Cambridge, U.K.: Cambridge Univ. Press, 2008.

[7] R. Zekevat and R. Buehrur, Wireless Localization Using Ultra-Wideband Signals, 1st ed. New York, NY, USA: Wiley, 2012.

[8] D. Dardari, A. Conti, U. Ferner, A. Giorgetti, and M. Z. Win, "Ranging with ultrawide bandwidth signals in multipath environments," Proc. IEEE, vol. 97, no. 2, pp. 404-426, Feb. 2009.

[9] S. Gezici et al., "Localization via ultra-wideband radios: A look at positioning aspects for future sensor networks," IEEE Signal Process. Mag., vol. 22, no. 4, pp. 70-84, Jul. 2005.

[10] B. Sobhani, M. Mazzotti, E. Paolini, A. Giorgetti, and M. Chiani, "Effect of state space partitioning on Bayesian tracking for UWB radar sensor networks," in Proc. IEEE Int. Conf. Ultra-Wideband (ICUWB), Sep. 2013, pp. 120-125.

[11] B. Sobhani, E. Paolini, A. Giorgetti, M. Mazzotti, and M. Chiani, "Bayesian tracking in UWB radar sensor networks," in Proc. IEEE Int. Conf. Commun. Workshops (ICC), Jun. 2013, pp. 47-51.

[12] S. Bartoletti, A. Conti, and A. Giorgetti, "Analysis of UWB radar sensor networks," in Proc. IEEE Int. Conf. Commun. (ICC), May 2010, pp. 1-6.

[13] M. Švecová, D. Kocur, R. Zetik, and J. Rovnakova, "Target localization by a multistatic UWB radar," in Proc. 20th Int. Conf. Radioelektronika (RADIOELEKTRONIKA), Apr. 2010, pp. 1-4.
[14] X.-W. Zhao, A. Gaugue, C. Lièbe, J. Khamlichi, and M. Menard, "Through the wall detection and localization of a moving target with a bistatic UWB radar system," in Proc. Eur. Radar Conf. (EuRAD), Sep. 2010, pp. 204-207.

[15] G. Wei, Y. Zhou, and S. Wu, "Detection and localization of high speed moving targets using a short-range UWB impulse radar," in Proc. IEEE Radar Conf. (RADAR), May 2008, pp. 1-4.

[16] D.-H. Kim, D.-W. Lim, L. Shen, H.-M. Kim, S.-C. Woo, and H. K. Yu, "Localization methods of multi-targets for UWB radar sensor networks," in Proc. 3rd Int. Asia-Pacific Conf. Synth. Aperture Radar (APSAR), Sep. 2011, pp. 1-4.

[17] H. Ding, C. Chen, S. Peng, X. Li, and L. Zheng, "Multistatic ultrawideband localization for NLOS environments," in Proc. 2nd Int. Conf. Intell. Syst. Design Eng. Appl. (ISDEA), Jan. 2012, pp. 380-384.

[18] S. Balckman and R. Popoli, Design and Analysis of Modern Tracking Systems. Norwood, MA, USA: Artech House, 2010.

[19] R. P. S. Mahler, Statistical Multisource-Multitarget Information Fusion. Norwood, MA, USA: Artech House, 2007.

[20] R. P. S. Mahler, "Multitarget Bayes filtering via first-order multitarget moments," IEEE Trans. Aerosp. Electron. Syst., vol. 39, no. 4, pp. 1152-1178, Oct. 2003.

[21] M. S. Arulampalam, S. Maskell, N. Gordon, and T. Clapp, "A tutorial on particle filters for online nonlinear/non-Gaussian Bayesian tracking," IEEE Trans. Signal Process., vol. 50, no. 2, pp. 174-188, Feb. 2002.

[22] B. Ristic, S. Arulampalam, and N. Gordon, Beyond the Kalman Filter: Particle Filters for Tracking Applications. Norwood, MA, USA: Artech House, 2007.

[23] F. Gustafsson, "Particle filter theory and practice with positioning applications," IEEE Aerosp. Electron. Syst. Mag., vol. 25, no. 7, pp. 53-82, Jul. 2010.

[24] S. Jovanoska and R. Thoma, "Multiple target tracking by a distributed UWB sensor network based on the PHD filter," in Proc. 15th Int. Conf. Inf. Fusion (FUSION), Jul. 2012, pp. 1095-1102.

[25] B. Sobhani, E. Paolini, A. Giorgetti, M. Mazzotti, and M. Chiani, "Target tracking for UWB multistatic radar sensor networks," IEEE J. Sel. Topics Signal Process., vol. 8, no. 1, pp. 125-136, Feb. 2014.

[26] S. Jovanoska, R. Zetik, R. Thoma, F. Govaers, K. Wilds, and W. Koch, "Device-free indoor localization using a distributed network of autonomous UWB sensor nodes," in Proc. Workshop Sensor Data Fusion, Trends, Solutions, Appl. (SDF), Oct. 2013, pp. 1-6.

[27] Y. Kilic, H. Wymeersch, A. Meijerink, M. J. Bentum, and W. G. Scanlon, "Device-free person detection and ranging in UWB networks," IEEE J. Sel. Topics Signal Process., vol. 8, no. 1, pp. 43-54, Feb. 2014.

[28] Y. Zhou, C. L. Law, Y. L. Guan, and F. Chin, "Localization of passive target based on UWB backscattering range measurement," in Proc. IEEE Int. Conf. Ultra-Wideband (ICUWB), Sep. 2009, pp. 145-149.

[29] Time Domain. [Online]. Available: http://www.timedomain.com/, accessed Jun. 2014

[30] X. R. Li and V. P. Jilkov, "Survey of maneuvering target tracking. Part I. Dynamic models," IEEE Trans. Aerosp. Electron. Syst., vol. 39, no. 4, pp. 1333-1364, Oct. 2003.

[31] Y. Bar-Shalom, X. R. Li, and T. Kirubarajan, Estimation With Applications to Tracking and Navigation. New York, NY, USA: Wiley, 2001.

[32] A. F. Cattoni, A. Dore, and C. S. Regazzoni, "Video-radio fusion approach for target tracking in smart spaces," in Proc. IEEE Int. Conf. Inf. Fusion (FUSION), Jul. 2007, pp. 1-7.

[33] A. Kushki, K. N. Plataniotis, and A. N. Venetsanopoulos, "Intelligent dynamic radio tracking in indoor wireless local area networks," IEEE Trans. Mobile Comput., vol. 9, no. 3, pp. 405-419, Mar. 2010.

[34] Y.-S. Chiou, C.-L. Wang, and S.-C. Yeh, "An adaptive location estimator based on Kalman filtering for dynamic indoor environments," in Proc. IEEE Veh. Technol. Conf. (VTC), Sep. 2006, pp. 1-5.

[35] B.-N. Vo and W.-K. Ma, "The Gaussian mixture probability hypothesis density filter," IEEE Trans. Signal Process., vol. 54, no. 11, pp. 4091-4104, Nov. 2006.

[36] B.-N. Vo, S. Singh, and A. Doucet, "Sequential Monte Carlo methods for multitarget filtering with random finite sets," IEEE Trans. Aerosp. Electron. Syst., vol. 41, no. 4, pp. 1224-1245, Oct. 2005.

[37] B. Ristic, D. Clark, and B.-N. Vo, "Improved SMC implementation of the PHD filter," in Proc. IEEE Int. Conf. Inf. Fusion (FUSION), 2010, pp. $1-8$.

[38] E. Ozkan, M. B. Guldogan, U. Orguner, and F. Gustafsson, "Ground multiple target tracking with a network of acoustic sensor arrays using PHD and CPHD filters," in Proc. IEEE Int. Conf. Inf. Fusion (FUSION), Jul. 2011, pp. 1-8. 
[39] B.-N. Vo and W.-K. Ma, "The Gaussian mixture probability hypothesis density filter," IEEE Trans. Signal Process., vol. 54, no. 11, pp. 4091-4104, Nov. 2006.

[40] D. Clark, B.-T. Vo, B.-N. Vo, and S. Godsill, "Gaussian mixture implementations of probability hypothesis density filters for non-linear dynamical models," in Proc. IET Seminar Target Tracking \& Data Fusion, Algorithms Appl., Apr. 2008, p. 19.

[41] M. Lei, Z. Jing, and P. Dong, "Extended GM-PHD filter for multitarget tracking in nonlinear/non-Gaussian system," in Proc. IEEE 17th Int. Conf. Inf. Fusion (FUSION), Jul. 2014, pp. 1-8.

[42] M. B. Guldogan, D. Lindgren, F. Gustafsson, H. Habberstad, and U. Orguner, "Multi-target tracking with PHD filter using Doppler-only measurements," Digital Signal Process., vol. 27, pp. 1-11, Apr. 2014

[43] M. B. Guldogan, U. Orguner, and F. Gustafsson, "Gaussian mixture PHD filter for multi-target tracking using passive Doppler-only measurements," in Proc. 9th IET Data Fusion Target Tracking Conf., Algorithms Appl., May 2012, pp. 1-6.

[44] D. Clark, B.-N. Vo, and J. Bell, "GM-PHD filter multitarget tracking in sonar images," Proc. SPIE, vol. 6235, p. 62350R, May 2006.

[45] M. Ulmke, O. Erdinc, and P. Willett, "GMTI tracking via the Gaussian mixture cardinalized probability hypothesis density filter," IEEE Trans. Aerosp. Electron. Syst., vol. 46, no. 4, pp. 1821-1833, Oct. 2010.

[46] D. Laneuville and J. Houssineau, "Passive multi target tracking with GM-PHD filter," in Proc. 13th IEEE Int. Conf. Inf. Fusion (FUSION), Jul. 2010, pp. 1-7.

[47] R. Georgescu and P. Willett, "The GM-CPHD tracker applied to real and realistic multistatic sonar data sets," IEEE J. Ocean. Eng., vol. 37, no. 2, pp. 220-235, Apr. 2012.

[48] M. Pace, "Comparison of PHD based filters for the tracking of 3D aerial and naval scenarios," in Proc. IEEE Radar Conf., May 2010, pp. $479-484$.

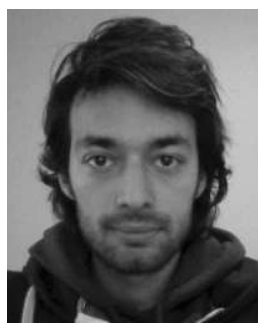

Berk Gulmezoglu was born in Turkey in 1990 He received the B.S. and M.S. degrees in electrical and electronics engineering from Bilkent University, Ankara, Turkey, in 2012 and 2014, respectively. He is currently pursuing the Ph.D. degree at the Worcester Polytechnic Institute, Worcester, MA, USA. His research interests are detection and estimation, signal processing, UWB systems, cryptography, and sidechannel attacks.

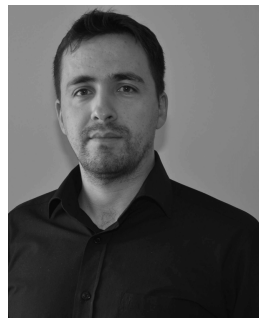

Mehmet Burak Guldogan received the B.S., M.S., and $\mathrm{Ph} . \mathrm{D}$. degrees in electrical and electronics engineering from Bilkent University, Ankara, Turkey, in 2003, 2006, and 2010, respectively. From 2010 to 2012, he was a Post-Doctoral Fellow with the Automatic Control Group, Linköping University, Linköping, Sweden. He joined Turgut Özal University, Ankara, in 2012, where he is currently an Assistant Professor with the Department of Electrical and Electronics Engineering. He has been an Associate Editor of Digital Signal Processing (Elsevier) since 2012. His current research interests are in statistical signal processing, sensor fusion, estimation theory, and target tracking.

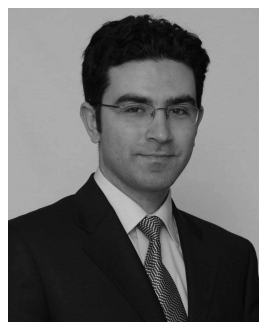

Sinan Gezici (S'03-M'06-SM'11) received the B.S. degree from Bilkent University, Ankara, Turkey, in 2001, and the Ph.D. degree in electrical engineering from Princeton University, Princeton, NJ, USA, in 2006. From 2006 to 2007, he was with Mitsubishi Electric Research Laboratories, Cambridge, MA, USA. Since 2007, he has been with the Department of Electrical and Electronics Engineering, Bilkent University, where he is currently an Associate Professor.

Dr. Gezici's research interests are in the areas of detection and estimation theory, wireless communications, and localization systems. Among his publications in these areas is the book entitled Ultrawideband Positioning Systems: Theoretical Limits, Ranging Algorithms, and Protocols (Cambridge University Press, 2008). He is an Associate Editor of the IEEE TRANSACTIONS ON COMMUNications, the IEEE Wireless COMMUNiCATIONS LETTERs, and the Journal of Communications and Networks. 